OPEN ACCESS

Edited by:

Amanda Sferruzzi-perri, University of Cambridge,

United Kingdom

Reviewed by:

Mark Robert Dilworth,

The University of Manchester,

United Kingdom

Adam John Watkins,

University of Nottingham,

United Kingdom

Robin Gandley,

Magee-Womens Research Institute,

United States

${ }^{*}$ Correspondence: Kirsty G. Pringle

kirsty.pringle@newcastle.edu.au

Specialty section:

This article was submitted to Embryonic and Developmental

Physiology,

a section of the journal

Frontiers in Physiology

Received: 03 August 2020 Accepted: 09 September 2020 Published: 30 September 2020

Citation:

Tamanna S, Clifton VL, Rae K, van Helden DF, Lumbers ER and

Pringle KG (2020) Angiotensin

Converting Enzyme 2 (ACE2)

in Pregnancy: Preeclampsia

and Small for Gestational Age.

Front. Physiol. 11:590787.

doi: 10.3389/fphys.2020.590787

\section{Angiotensin Converting Enzyme 2 (ACE2) in Pregnancy: Preeclampsia and Small for Gestational Age}

\author{
Sonia Tamanna ${ }^{1,2,3}$, Vicki L. Clifton ${ }^{4,5}, K_{y m}$ Rae $^{5}$, Dirk F. van Helden ${ }^{2}$, \\ Eugenie R. Lumbers ${ }^{1,2,3}$ and Kirsty G. Pringle ${ }^{1,2,3 *}$
}

${ }^{1}$ Priority Research Centre for Reproductive Sciences, University of Newcastle, Newcastle, NSW, Australia, ${ }^{2}$ School of Biomedical Sciences and Pharmacy, Faculty of Health and Medicine, University of Newcastle, Newcastle, NSW, Australia, ${ }^{3}$ Pregnancy and Reproduction Program, Hunter Medical Research Institute, University of Newcastle, Newcastle, NSW, Australia, ${ }^{4}$ School of Medicine, Robinson Research Institute, University of Adelaide, Adelaide, SA, Australia, ${ }^{5}$ Mater Medical Research Institute and Translational Research Institute, University of Queensland, Brisbane, QLD, Australia

Introduction: An imbalance in angiotensin (Ang) peptides could contribute to the pathophysiology of preeclampsia (PE) and poor fetal growth.

Methods: We measured maternal plasma levels of Ang peptides and converting enzymes in non-pregnant women $(n=10)$, in normal pregnant women $(n=59)$, women delivering small for gestational age babies (SGA, $n=25$ ) across gestation (13-36 weeks) and in women with PE $(n=14)$ in their third trimester.

Results: Plasma ACE, ACE2, and Ang-(1-7) levels, and ACE2 activity were significantly higher in normal pregnant women compared with non-pregnant women; neprilysin (NEP) levels were not changed. In SGA pregnancies, ACE and ACE2 levels were higher in early-mid pregnancy compared with normal pregnant women. In women with PE, plasma ACE, ACE2, NEP, and Ang-(1-7) levels and ACE2 activity were lower than levels in normal pregnant women.

Conclusion: The higher plasma ACE2 levels and activity in pregnancy could be driving the higher Ang-(1-7) levels. The early gestation increases in ACE and ACE2 levels in SGA pregnancies highlights the possibility that these enzymes could be used as potential early biomarkers of poor fetal growth. In women with PE, the reduced ACE2 and NEP levels at term, could be contributing to the reduction in Ang-(1-7) levels. These findings suggest that dysfunctional relationships between two key enzymes in the circulating RAS are involved in the pathogenesis of PE and SGA. Since soluble ACE2 can prevent binding of the novel coronavirus, SARS-CoV-2, to membrane bound ACE2, the interplay between ACE2 and the coronavirus and its impact in pregnancy requires further investigation.

Keywords: angiotensin converting enzyme 2 (ACE2), angiotensin peptides, preeclampsia, pregnancy, small for gestational age 


\section{INTRODUCTION}

During pregnancy, the renin-angiotensin system (RAS) plays a significant role in the regulation of blood pressure and salt and water balance. It is activated by estrogen-induced increases in angiotensinogen (AGT) and, subsequently, by the reduction in systemic vascular resistance caused by the ovarian hormone, relaxin (Lumbers and Pringle, 2014). It is perhaps not surprising then that alterations in the circulating and intrauterine RAS are associated with the development of preeclampsia $(\mathrm{PE})$ and pregnancies in which the infant is born small for gestational age (SGA) (Irani and Xia, 2008; Zhou et al., 2013; Delforce et al., 2019).

The circulating RAS cascade (Figure 1) begins with the release of active renin from the kidney, which cleaves angiotensin I (Ang I) from AGT. Angiotensin II (Ang II), the major effector molecule of the RAS cascade, is cleaved from Ang I by angiotensin converting enzyme (ACE). Ang II elicits its effects by binding to the Ang II type 1 receptor $\left(\mathrm{AT}_{1} \mathrm{R}\right)$. Interactions between Ang II and the $\mathrm{AT}_{1} \mathrm{R}$ stimulate sodium retention (both directly and via stimulating the release of aldosterone) and raise blood pressure, both by central actions and direct effects on blood vessels. Alternatively, Ang II can bind to the $\mathrm{AT}_{2} \mathrm{R}$, which has opposing actions than those of Ang II binding to the $\mathrm{AT}_{1} \mathrm{R}$ (Escobar et al., 2004).

The actions of Ang II/AT $R$ are counter-balanced by an alternate RAS axis (Figure 1). This axis encompasses ACE2, which can form Ang-(1-7) by removing a single amino acid from Ang II. In conjunction with ACE, ACE2 can also form Ang-(1-7) from Ang I, as can neprilysin (NEP) by acting on Ang I. Ang(1-7) acting through the Mas receptor, opposes the actions of the Ang II/ $/ \mathrm{AT}_{1} \mathrm{R}$ pathway, as do interactions between Ang II and the $\mathrm{AT}_{2} \mathrm{R}$ (Warner et al., 2004).

ACE2 plays a role in balancing the vasoconstrictor and vasodilator arms of the RAS (Gallagher et al., 2008) and exerts a protective role in end organ damage (heart, lung, kidney, etc.) (Oudit et al., 2009; Wang et al., 2016). ACE2 can undergo ectodomain shedding to secrete a soluble form of ACE2, sACE2 (Lambert et al., 2005).

ACE2 has an almost 500-fold higher catalytic efficiency in forming Ang-(1-7) from Ang II than in converting Ang I to Ang(1-9) (Vickers et al., 2002), which is then cleaved by ACE. ACE2 is insensitive to ACE inhibitors such as captopril, lisinopril, and enalapril (Donoghue et al., 2000), and there is evidence that ACE2 counteracts the effects of the RAS mediated by over activity of Ang II and the $\mathrm{AT}_{1} \mathrm{R}$ (Chhabra et al., 2013).

Plasma Ang-(1-7) is increased in pregnancy, whereas ACE concentrations are decreased (Merrill et al., 2002). In women with PE, maternal plasma Ang-(1-7), Ang II and plasma renin activity are reduced compared with normotensive pregnant women (Merrill et al., 2002; Velloso et al., 2007). ACE concentrations are however, increased in women with $\mathrm{PE}$ (Merrill et al., 2002). Women who gave birth to SGA babies also had higher concentrations of plasma ACE at 15 weeks' gestation compared with women who delivered babies of normal birth weight (Zhou et al., 2013). To the best of our knowledge, there are no data on changes in maternal ACE2 and NEP levels in either normal pregnant women or women suffering from complicated pregnancies. It is possible for Ang-(1-7) to be formed via the activity of both these pathways (see Figure 1).

ACE2 is the critical receptor for the highly pathogenic novel SARS-CoV-2 virus, which causes COVID-19. The SARS-CoV-2 spike protein binds with human ACE2 with substantially higher affinity than does the spike protein of SARS-CoV (Walls et al., 2020; Wan et al., 2020; Wrapp et al., 2020). Therefore, our particular interest in measuring sACE2 in pregnant women could be important in understanding the pathogenesis of SARS-CoV2 in pregnancy.

In this study, we measured ACE, sACE2, Ang-(1-7) and NEP levels in plasma from women with uncomplicated (normal) pregnancies and compared them to levels found in healthy non-pregnant women. We also measured plasma levels of these components of the RAS in women with pregnancies complicated by either PE or SGA.

\section{MATERIALS AND METHODS}

\section{Study Population}

Data and biobanked plasma samples from a cohort study undertaken at the Lyell McEwin Hospital, Adelaide, Australia, that was used to study the effects of asthma during pregnancy on the mother, placenta and baby, were examined (Dickinson et al., 2016). The study was approved by the Queen Elizabeth Hospital and Lyell McEwin Hospital Human Research Ethics Committee and the University of Adelaide Human Research Ethics Committee. Samples were collected from $(n=59)$ women with uncomplicated pregnancies at $13,18,30$, and 36 weeks. Samples were also collected from 25 women with SGA pregnancies $(13,18,30$, and 36 weeks) and 5 women who developed PE (range: 30-36 weeks).

We used stored plasma samples from healthy non-pregnant women $(n=10)$, and women who developed preeclampsia ( $n=9,26-35$ weeks of gestation, Supplementary Table 1) collected at the John Hunter Hospital and Tamworth Base Hospital, NSW, Australia. The collection and use of these samples were approved by the University of Newcastle Research Ethics Committee and Hunter New England Health Human Research Ethics Committee. The normal pregnancy group included individuals who delivered appropriately grown infants $\left(>10^{\text {th }}\right.$ centile for gestational age) at term ( $>37$ weeks) who did not have hypertension, diabetes, infections, or renal dysfunction. Women were classified as having PE based on the SOMANZ definition (Lowe et al., 2015). However, some samples were collected prospectively, at which time we measured blood pressure. Pregnancies complicated by SGA had babies whose birth weights were $<10^{\text {th }}$ centile for gestational age calculated using the GROW (Gestation Related Optimal Weight) method (https://www.gestation.net/).

\section{Blood Sampling}

For all samples, maternal venous blood was collected in lithium/heparin tubes, centrifuged at $1000 \mathrm{~g}$ for $15 \mathrm{~min}$, aliquoted and stored at $-80^{\circ} \mathrm{C}$ until assays were performed. 


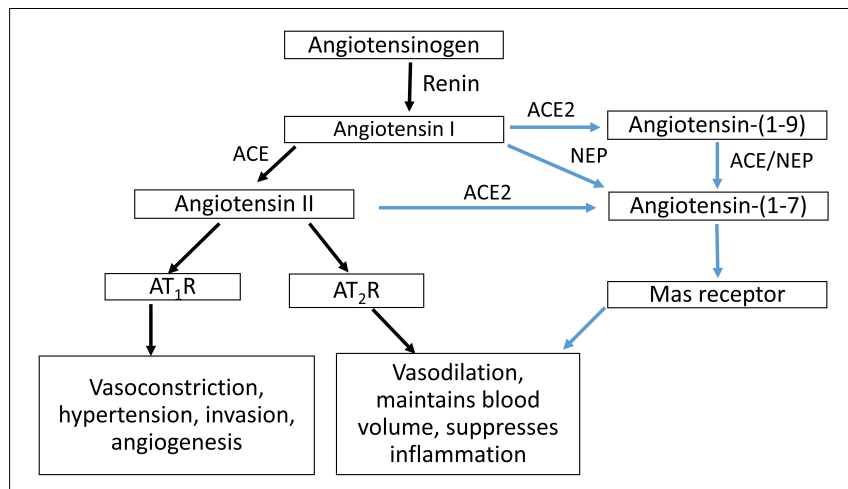

FIGURE 1 | Schematic representation of the RAS cascade. ACE, angiotensin converting enzyme; ACE2, angiotensin converting enzyme 2; NEP, neprilysin; $\mathrm{AT}_{1} \mathrm{R}$, Angiotensin II type 1 receptor, $\mathrm{AT}_{2} \mathrm{R}$; Angiotensin II type 2 receptor.

\section{Measurement of Plasma ACE, ACE2, and NEP Levels}

Maternal plasma ACE and NEP concentrations were determined using commercially available enzyme-linked immunosorbent assay kits according to the instructions of the manufacturer (Duoset, R\&D Systems, Minneapolis, MN, United States). Concentrations of maternal plasma ACE2 were measured using commercial enzyme-linked immunosorbent assay (ELISA) kits (Cloud-Clone Corp, Houston, TX, United States). All samples were assayed in duplicate. The mean intra- and inter-assay coefficients of variations were $6.88 \%(n=131)$ and $15.16 \%(5$ plates) for ACE, $4.68 \%(n=131)$ and $13.22 \%$ (6 plates) for ACE2, and $7.89 \%(n=96)$ and $1.24 \%$ for NEP (4 plates), respectively.

\section{Determination of ACE2 Activity}

The ACE2 activity assay was carried out according to a published protocol with minor modifications (Shao et al., 2013; Xiao and Burns, 2017). Briefly, $15 \mu \mathrm{L}$ of each plasma sample was diluted with an enzyme buffer consisting of $1 \mathrm{M} \mathrm{NaCl}, 75 \mathrm{mM}$ Tris$\mathrm{HCl}$, $5.0 \mathrm{mM} \mathrm{ZnCl}$, pH 6.5 with protease inhibitors, which were $10 \mu \mathrm{M}$ captopril, $5 \mu \mathrm{M}$ amastatin, and $10 \mu \mathrm{M}$ bestatin (all from Sigma Aldrich, St. Louis, Missouri, United States) and $10 \mu \mathrm{M}$ Z-prolyl-prolinal (Enzo Life Sciences, Inc., NY, United States). The ACE2-specific quenched fluorescent substrate, MCA-AlaPro-Lys-2, 4 dinitrophenyl (AnaSpec, Inc., San Diego, CA, United States) diluted in enzyme buffer were added to the samples in a final concentration of $50 \mu \mathrm{M}$ in $100 \mu \mathrm{L}$ on a black 96-well microplate. The plate was covered with aluminum foil to protect from light and incubated at room temperature for $24 \mathrm{~h}$ on a plate shaker. We have used recombinant human ACE2 (R\&D Systems, Minneapolis MN) as the standard instead of using a fluorescent product to measure ACE2 activity. ACE2 cleaves the Pro-Lys bond of the substrate, and the Relative Fluorescence Units (RFU) of the released Mca-Ala-Pro was measured over a period of $24 \mathrm{~h}$. The plate was read on a fluorescence reader (Clario Star, BMG Labtech, GmbH, Ortenberg, Germany) with an excitation wavelength of $320 \mathrm{~nm}$ and emission wavelength of $405 \mathrm{~nm}$. The specificity of the enzyme activity was checked using $1 \mu \mathrm{M}$ of an ACE2 inhibitor (DX600; AnaSpec, Inc., San
Diego, CA, United States), which is specific for human ACE2. We calculated how much ACE2 activity there was in a sample comparing the RFU to the RFU of known concentrations of recombinant $\mathrm{ACE} 2$, that were treated in an identical manner. Thus ACE2 activity is reported as the activity $/ \mathrm{ng} / \mathrm{ml}$ of ACE2. All samples were assayed in duplicate. The mean intra and interassay coefficients of variations were $7.17 \%(n=136)$ and $4.16 \%(5$ plates), respectively.

\section{Measurement of Angiotensin-(1-7)}

A direct radioimmunoassay (RIA) was used to measure plasma Ang-(1-7) levels by Prosearch Pty Ltd (Malvern, VIC, Australia) as described previously (Sykes et al., 2014b).

\section{Statistical Analyses}

Data for all RAS enzyme levels and activity, as well as Ang-(17) levels, ACE2/ACE ratio, and ACE2 activity/ACE2 ratio, were expressed as median and interquartile ranges. A non-parametric Kruskal-Wallis test (with Dunn's multiple comparison test) was performed to compare ACE, ACE2, NEP and Ang-(1-7) levels and ACE2 activity, ACE2/ACE ratio, and ACE2 activity/ACE2 ratio between non-pregnant women and women with normal pregnancies (at 13, 18, 30, and 36 weeks of gestation). Mixed effect analysis (with Fisher's LSD multiple comparison test) was used to compare differences between normal pregnancies and pregnancies complicated by SGA at each gestational age. A Mann-Whitney test was used to determine differences between normal pregnancies and women with PE in the third trimester. Spearman's correlations were used to determine any association between ACE2 and Ang-(1-7) levels and ACE2 levels as well as birth weight centile. Differences in demographic characteristics of different pregnant groups were identified by the chi-square test. Differences were considered significant if $P<0.05$.

\section{RESULTS}

\section{Baseline Characteristics of the Study Population}

A total of 10 non-pregnant women were recruited in this study. The median age was 26.5 years (interquartile range (IQR): $25-$ 35 years) and BMI was $25.32 \mathrm{~kg} / \mathrm{m}^{2}$ (IQR: 22.83-28.30). Three of the ten non-pregnant women were taking oral contraceptives, and a further four were using hormonal contraceptive devices (e.g., Implanon and Mirena).

Table 1 outlines the baseline characteristics of each pregnancy group. Maternal age, BMI and ethnicity were not significantly different between the study groups. The median birth weights and birth weight centiles of SGA infants were less $(P<0.001$ and $P<0.001$, respectively), as were the birth weights and birth weight centiles of infants whose mothers had PE $(P<0.001$ and $P=0.002$, respectively) than those from normal pregnancies. Women with PE delivered at an earlier gestational age compared with those who had normal pregnancies $(\mathrm{P}<0.001)$, which could account for their lower birth weights. No significant difference was observed in the gestational age at delivery of 
TABLE 1 | Baseline characteristics of normal, PE and SGA pregnancies.

\begin{tabular}{|c|c|c|c|}
\hline & $\begin{array}{c}\text { Normal } \\
\text { Pregnancy } \\
n=59\end{array}$ & PE $n=14$ & SGA $n=25$ \\
\hline $\begin{array}{l}\text { Maternal age } \\
\text { (years) }\end{array}$ & $26(23-30.3)$ & $26.6(22.8-29.0)$ & $27.5(19-31)$ \\
\hline BMI $\left(k g / m^{2}\right)$ & $26.6(23.1-30.4)$ & $27.97(24.9-32.4)$ & $29.8(25.7-34.2)$ \\
\hline $\begin{array}{l}\text { GA at delivery } \\
\text { (weeks) }\end{array}$ & $39.6(38.4-40.3)$ & $\begin{array}{c}35.53 \\
(33.8-38.0)^{*}\end{array}$ & 39.7 (38.2-40.9) \\
\hline Birth weight (g) & $\begin{array}{c}3460 \\
(3140-3735)\end{array}$ & $\begin{array}{c}2395 \\
(1553-2908)^{\star}\end{array}$ & $\begin{array}{c}2830 \\
(2543-3080)^{\star}\end{array}$ \\
\hline BWC & $46.40(27.6-75.0)$ & $9.2(0.4-56.4)^{*}$ & $5.25(1.8-7.2)^{\star}$ \\
\hline \multicolumn{4}{|l|}{$\begin{array}{l}\text { Maternal } \\
\text { ethnicity }\end{array}$} \\
\hline Caucasian & $43(72.88)$ & $12(85.71)$ & $20(80)$ \\
\hline Not Caucasian & $16(27.12)$ & $2(14.29)$ & $5(20)$ \\
\hline Smoker & $14(23.73)$ & 02 (14.29) & $12(48)^{\star}$ \\
\hline \multicolumn{4}{|l|}{ Infant sex } \\
\hline Male & $28(47.46)$ & $13(92.86)^{\star}$ & $10(40)$ \\
\hline Female & $31(52.54)$ & $1(7.14)$ & $15(60)$ \\
\hline \multicolumn{4}{|l|}{13 weeks } \\
\hline $\mathrm{sBP}(\mathrm{mmHg})$ & $110(105-115)$ & - & $111(109-125)$ \\
\hline $\mathrm{dBP}(\mathrm{mmHg})$ & 70 (60-70) & - & 70 (60-75) \\
\hline \multicolumn{4}{|l|}{18 weeks } \\
\hline sBP (mmHg) & 109 (100-120) & - & $112(110-121)$ \\
\hline $\mathrm{dBP}(\mathrm{mmHg})$ & $64(60-70)$ & - & 70 (70-80) \\
\hline \multicolumn{4}{|l|}{30 weeks } \\
\hline sBP (mmHg) & $112(102-120)$ & - & $117(106-123)$ \\
\hline $\mathrm{dBP}(\mathrm{mmHg})$ & $70(60-70)$ & - & 70 (60-80) \\
\hline \multicolumn{4}{|l|}{36 weeks } \\
\hline $\mathrm{sBP}(\mathrm{mmHg})$ & $110(100-120)$ & - & $110(100-129)$ \\
\hline $\mathrm{dBP}(\mathrm{mmHg})$ & $70(64-80)$ & - & $72(62-80)$ \\
\hline \multicolumn{4}{|l|}{$\begin{array}{l}\text { Third trimester } \\
\text { (26-36 weeks) }\end{array}$} \\
\hline $\mathrm{sBP}(\mathrm{mmHg})$ & $110(100-120)$ & 120 (110.5-136) & - \\
\hline $\mathrm{dBP}(\mathrm{mmHg})$ & $70(64-80)$ & $80(70-83)$ & - \\
\hline
\end{tabular}

Data are expressed as median (IQR) or number (\%) for maternal ethnicity, smoker, and infant sex. BMI, Body Mass Index; BWC, birth weight centile; dBP, diastolic blood pressure; GA, gestational age; PE, preeclampsia; sBP, systolic blood pressure; SGA, small for gestational age. *indicates a significant difference compared with the normal pregnancy group.

SGA babies compared with those born from women with normal pregnancies. The number of smokers was significantly higher in women delivering SGA babies compared to those with normal pregnancy outcomes $(P=0.028)$, whereas there was no significant differences between the prevalence of smokers between the PE and normal pregnancy groups. The number of male babies was higher $(P=0.002)$ in the $\mathrm{PE}$ group compared to normal pregnant group. Two of the women in the PE group were classified as having early-onset PE (developing $<34$ weeks gestation) and seven of the women delivered a baby who was SGA.

There were no significant differences in the systolic or diastolic blood pressures of women with normal pregnancies and those with SGA at any gestational age $(13,18,30$, or 36 weeks, Table 1). There were no differences in systolic and diastolic blood pressures in the PE group compared with the normal (normotensive) pregnancy group at the time of sampling in the third trimester (Table 1).

\section{Associations Between ACE2 Activity and ACE2 Levels as Well as Between ACE2 and Ang-(1-7) in Non-pregnant and Pregnant Women}

Figure 2 shows the association between plasma ACE2 activity and ACE2 levels as well as the association between plasma ACE2 and Ang-(1-7) in non-pregnant and pregnant women (normal, PE and SGA). ACE2 activity was positively correlated with ACE2 levels $(r=0.215, P=0.041)$. Plasma Ang-(1-7) levels were positively correlated with ACE2 levels and activity $(r=0.253, P=0.038$ and $r=0.333$, $P=0.028$, respectively).

\section{ACE, ACE2, NEP, and Ang-(1-7) Levels, ACE2 Activity and ACE2/ACE, ACE2 Activity/ACE2 Ratio in Normal Pregnancies}

Plasma ACE levels were significantly higher in women with normal pregnancies compared with non-pregnant women $(P<0.001)$ and remained high throughout gestation (Figure 3A). Women with normal pregnancies also had elevated ACE2 levels $(P=0.003)$ and activity $(P<0.001)$ compared with non-pregnant women (Figures 3B,C). However, ACE2 levels and activity did not change across gestation. NEP levels in non-pregnant and pregnant women were similar and did not change across gestation $(P=0.492$; Figure 3D).

In women with normal pregnancies, we determined if the known rise in plasma Ang II in pregnancy was counter-balanced by elevated Ang-(1-7) levels (Figure 3E). Plasma Ang-(1-7) levels were significantly increased in normal pregnant women compared with non-pregnant women $(P=0.001$, Figure 3E), this was statistically significant at all gestational ages examined except at 18 weeks of gestation.

We also examined the ACE2/ACE ratio (Figure 3F) as well as the ratio between ACE2 activity/ACE2 levels (Figure 3G) in normal pregnant women. In pregnancy, the ACE2/ACE ratio did not change across gestation $(P=0.243)$. However, the ACE2 activity/ACE2 ratio was significantly higher in normal pregnant women $(P=0.012)$ and remained elevated throughout gestation (Figure 3G).

\section{ACE, ACE2, NEP, and Ang-(1-7) Levels, ACE2 Activity and the ACE2/ACE and ACE2 Activity/ACE2 Ratios in SGA Pregnancies}

Women delivering babies who were born SGA had levels of ACE and ACE2 that were significantly higher than those in women with normal pregnancies $(P=0.048$ and $P<0.001$; Figures 4A,B). When examining the differences at each gestational age, the only statistical differences were at 18 weeks for ACE levels and at 13, 18, and 30 weeks for 


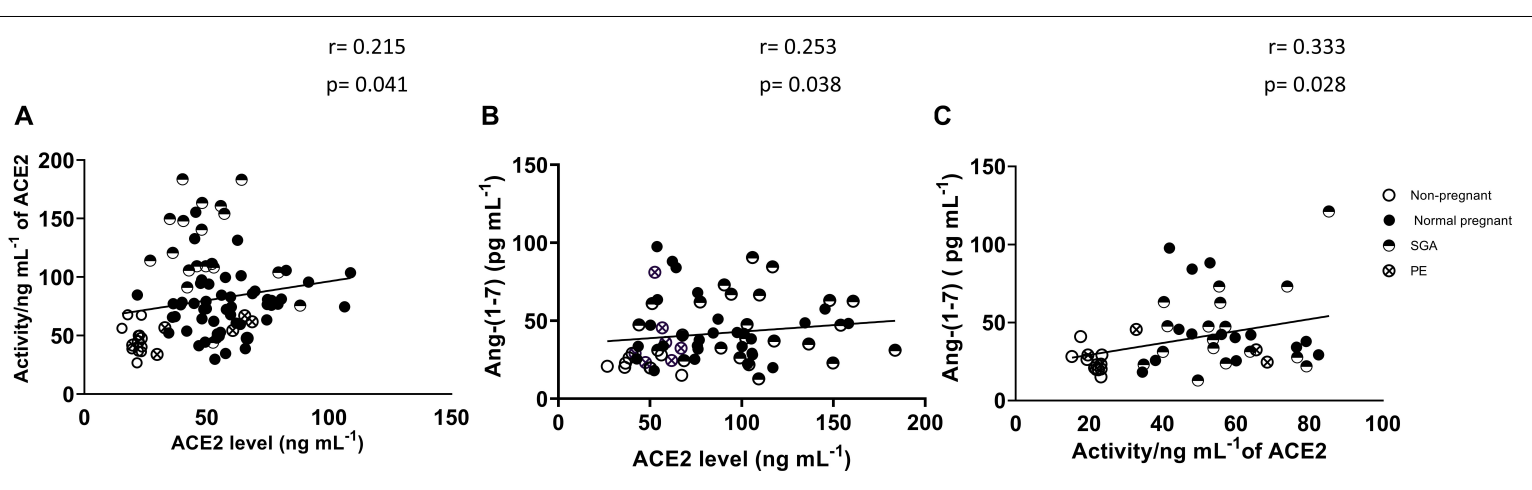

FIGURE 2 | Correlations between (A) ACE2 levels and ACE2 activity (non-pregnant and all pregnancy groups combined), (B) ACE2 levels and Ang-(1-7) levels, and (C) ACE2 activity and Ang-(1-7) levels.

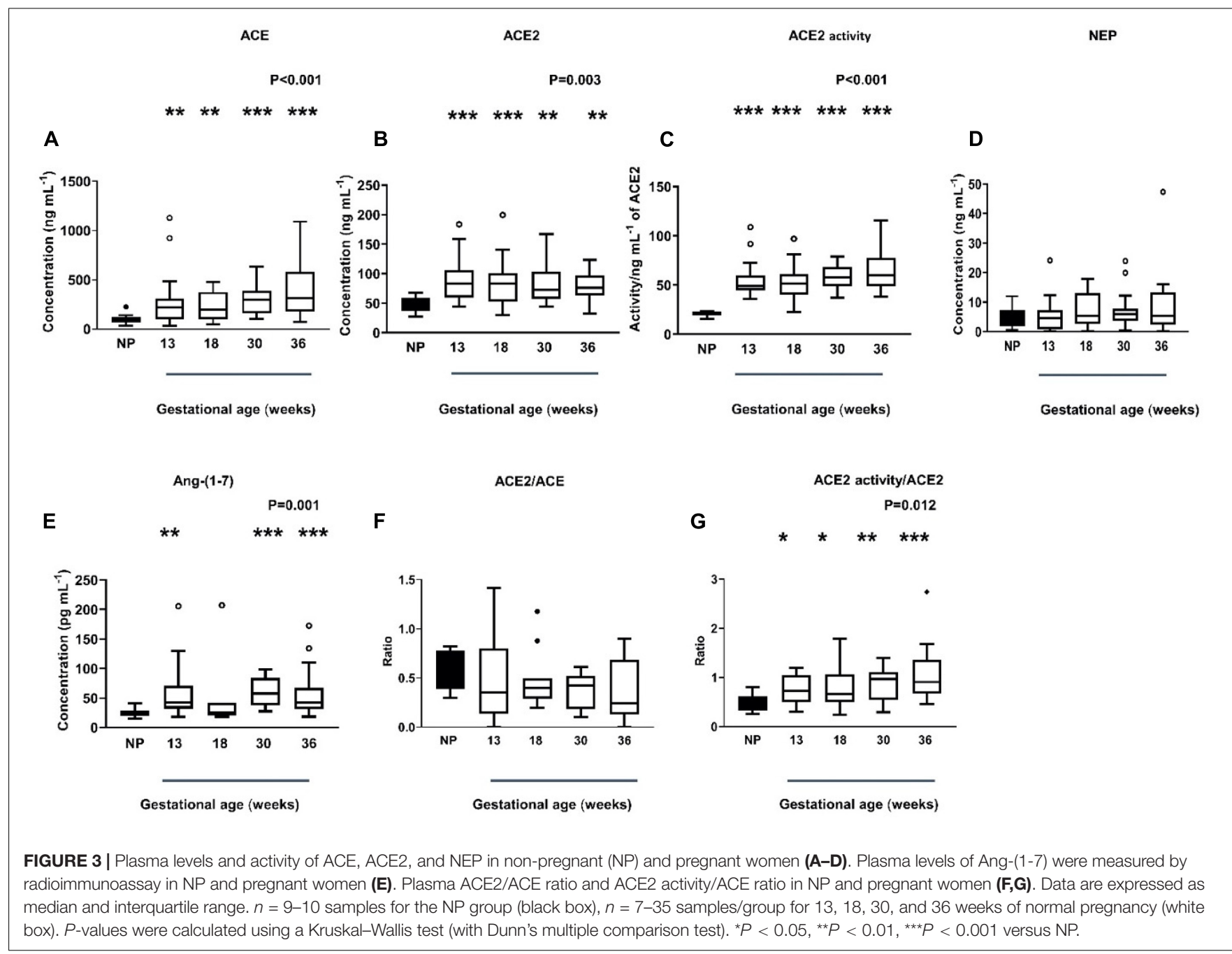

ACE2 levels. ACE2 activity however was not significantly different between normal and SGA pregnancies (Figure 4C). NEP and Ang-(1-7) levels in SGA pregnancies were similar to those found in women with normal pregnancies, as was the ACE2/ACE ratio (Figures 4D-F). However, the ratio between ACE2 activity and ACE2 levels was significantly decreased in women with SGA pregnancies compared with normal pregnancies $(P=0.005$; Figure 4G), this was significant at 13,18, and 30 weeks of gestation but not at 36 weeks. 

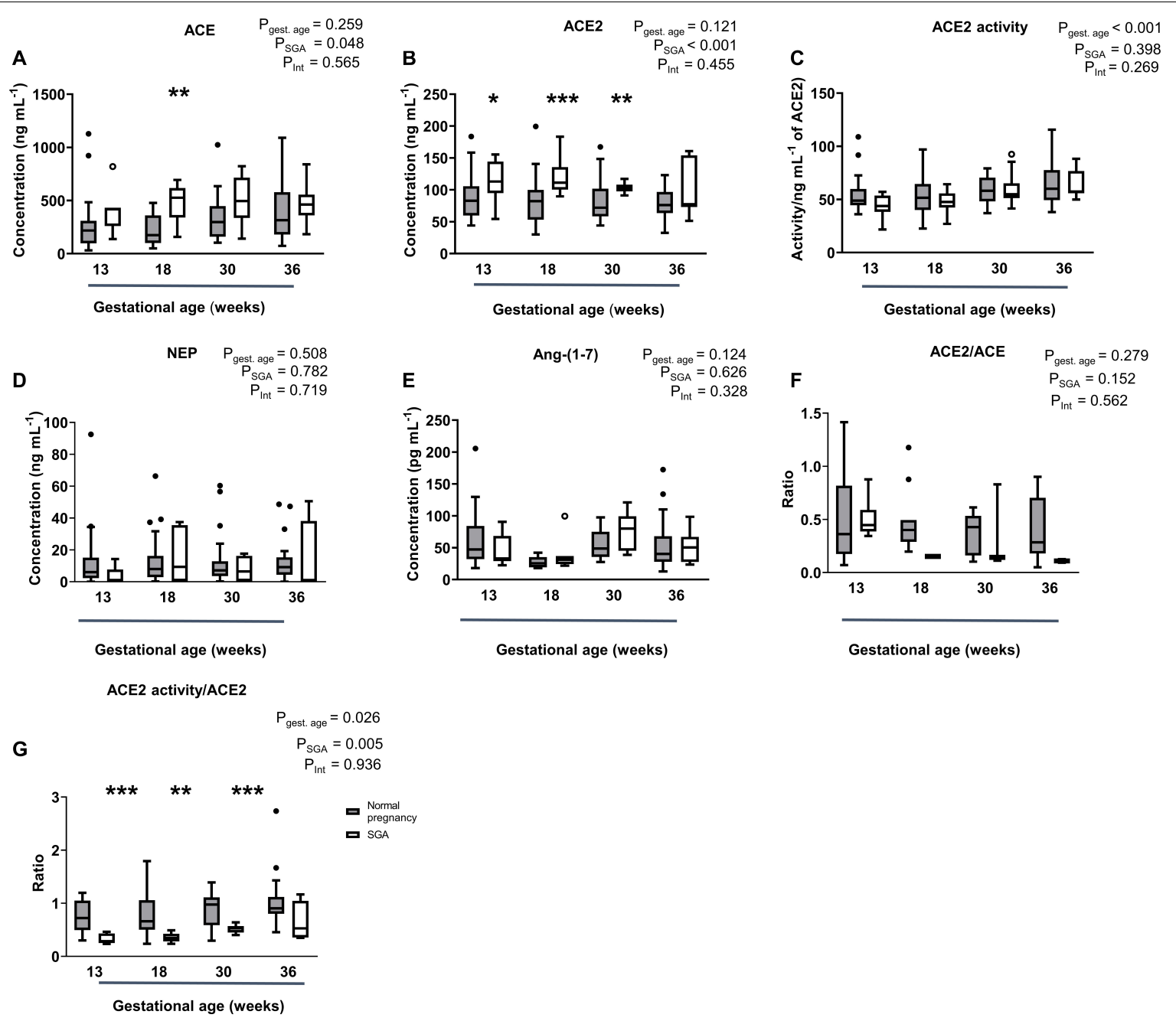

FIGURE 4 | Plasma levels and activity of (A) ACE, (B,C) ACE 2 , and (D) NEP as well as levels of (E) Ang-(1-7), (F) ACE2/ACE ratio, and (G) ACE2 activity/ACE2 ratio in normal and SGA pregnancies. Data are expressed as median and interquartile range. $n=5-36$ samples/group for normal pregnancies (gray box) and $n=4-15$ samples/group for SGA pregnancies (white box). P-values were calculated using a two-way ANOVA (mixed effect model with Fisher's LSD multiple comparison test). ${ }^{*} P<0.05,{ }^{* *} P<0.01,{ }^{* * *} P<0.001$ versus normal pregnancy group of the same gestational age.

\section{ACE, ACE2, NEP, AND ANG-(1-7) LEVELS, ACE2 ACTIVITY, AND THE ACE2/ACE RATIO IN WOMEN WITH PREECLAMPSIA}

ACE levels were significantly decreased in women with preeclampsia compared with levels measured in women with normal pregnancies $(P<0.001$; Figure 5A). Both plasma ACE2 levels $(P<0.001)$ and activity $(P=0.024)$ were significantly lower in women with preeclampsia compared with women with normal pregnancies (Figures 5B,C). There was no significant difference between the PE alone and PE with SGA samples in terms of ACE2 levels (data not shown). Plasma NEP levels were also decreased in women with $\mathrm{PE}(P=0.024$; Figure 5D).

Figure 5E shows the plasma Ang-(1-7) levels in women with normal pregnancies and women with PE. Women with PE had reduced levels of plasma Ang-(1-7) compared with levels in women with normal pregnancies $(P=0.034$; Figure $5 \mathrm{E})$. The ACE2/ACE ratio was increased in PE compared with normal pregnancies $(P<0.001$; Figure 5F).

\section{Associations Between ACE, ACE2, ACE2 Activity and Birth Weight Centile}

Table 2 shows the associations between ACE, ACE2 levels, and ACE2 activity and birth weight centile (all pregnant groups). Significant negative correlations were found between both ACE and ACE2 levels and birth weight centile (ACE: $r=-0.488$, $P=0.010, r=-0.384, P=0.044$ at 18 weeks and 30 weeks of gestation, ACE2: $r=-0.289, P=0.044, r=-0.627$, $P<0.001, r=-0.405, P=0.007$ for 13,18 , and 30 weeks of gestation; Table 2). ACE2 activity was not associated with birth weight centile. 

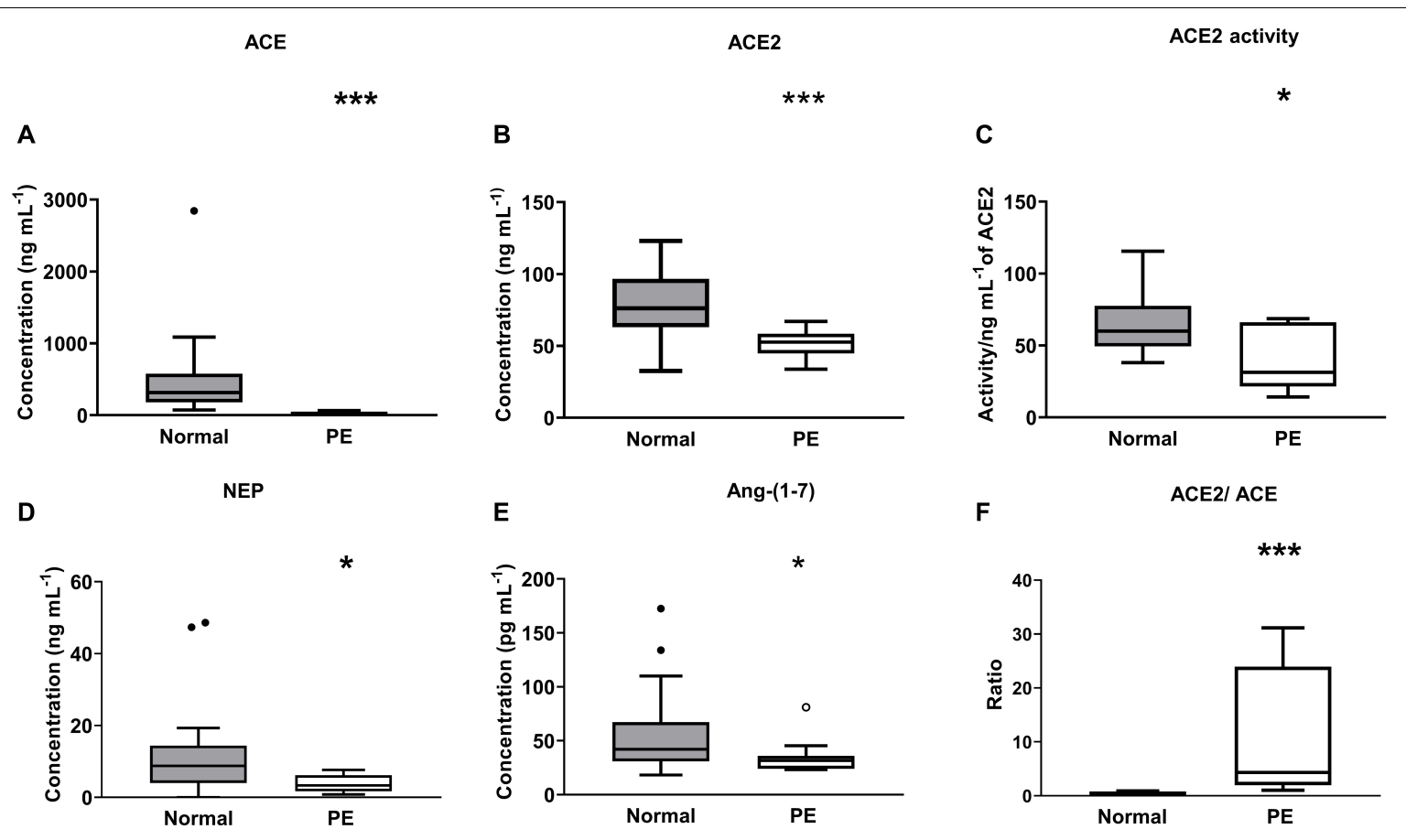

FIGURE $\mathbf{5}$ | Plasma levels and activity of (A) ACE, (B,C) ACE2, and (D) NEP as well as (E) levels of Ang-(1-7), and (F) ACE2/ACE ratio in the third trimester ( $\geq 26$ weeks of gestation) in women with normal pregnancies (gray box) and women with preeclampsia (PE; white box). Data are expressed as median and interquartile range. $n=16-32$ for normal pregnancy group and $n=8-14$ samples for the PE group. ${ }^{\star} P<0.05,{ }^{\star \star} P<0.01,{ }^{* \star \star} P<0.001$ versus normal pregnancy group.

TABLE 2 | Correlations between maternal SACE, SACE2, and SACE2 activity and birth weight centile (BWC).

\begin{tabular}{lcccc}
\hline & \multicolumn{1}{c}{ 13 weeks } & 18 weeks & 30 weeks & 36 weeks \\
\hline SACE vs. BWC & $r=-0.294 P=0.129$ & $r=-0.488 P=0.010^{\star}$ & $r=-0.338 P=0.044^{\star}$ & $r=-0.262 P=0.170$ \\
SACE2 vs. BWC & $r=-0.289 P=0.044^{\star}$ & $r=-0.627 P<0.001^{\star}$ & $r=-0.405 P=0.007^{\star}$ & $r=-0.013 P=0.936$ \\
SACE2 activity vs. BWC & $r=0.361 P=0.054$ & $r=0.050 P=0.789$ & $r=-0.041 P=0.826$ & $r=-0.175 P=0.404$ \\
\hline
\end{tabular}

Data show Spearman's correlation coefficient. *indicates significant correlations.

\section{DISCUSSION}

In this study, we showed for the first time that ACE and ACE2 levels and activity were increased in the maternal circulation during pregnancy and remained high throughout gestation, however NEP levels were unchanged. Plasma ACE2 levels were approximately $68 \%$ to $71 \%$ higher across gestation in women with normal pregnancies compared with non-pregnant women. We also confirmed that Ang-(1-7) levels were increased in pregnancy compared with non-pregnant women. In addition, we found that ACE2 levels were positively associated with ACE2 activity and that Ang-(1-7) levels were positively associated with both ACE2 levels and activity. This latter observation suggests that the amount and activity of ACE2 in the plasma is rate-limiting in terms of the production of Ang-(1-7). Whether or not this also applies to membrane bound ACE2 needs to be determined. The increased activity of ACE2 in normal pregnancy suggests that it may play a role, through the production of Ang-(1-7), in the regulation of maternal blood pressure. Previous studies carried out in ACE2 knockout mice show that ACE2 deficiency is associated with an increase in maternal blood pressure during pregnancy (Yamaleyeva et al., 2015). Several studies have also shown that polymorphisms in the ACE2 gene are associated with an increased risk of hypertension (Malard et al., 2013; Patnaik et al., 2014).

In women who gave birth to SGA neonates, circulating ACE levels were increased at 18 weeks of gestation compared with those found in women with normal pregnancies. Elevated levels of maternal plasma ACE have also been documented by Zhou et al. in women at 15 weeks' gestation who went on to deliver SGA neonates (Zhou et al., 2013). We found increased ACE2 levels in SGA pregnancies at 13,18 , and 30 weeks of gestation, but not in late pregnancy ( 36 weeks). It is possible, since ACE levels were increased at 18 weeks of gestation, that there was an elevation in ACE2 levels in early-mid gestation in order to regulate the activity of the Ang II via the $\mathrm{AT}_{1} \mathrm{R}$ pathway. Since ACE levels were not elevated at term, there was a corresponding reduction in ACE2 levels. ACE2 activity was not significantly different across gestation in women who later delivered SGA neonates compared with women with normal pregnancies. The increase in ACE2 
levels in the absence of any change in ACE2 activity could be explained by the presence of an endogenous inhibitor, which has been described in human plasma (Lew et al., 2008). Further studies are obviously required to investigate if this inhibitor is elevated in SGA pregnancies compared with normal pregnancies.

Interestingly, a negative correlation was found in early pregnancy between birth weight centiles and ACE and ACE2 levels (Table 2) suggesting that the increased production of angiotensin enzymes in early gestation could influence birth weight. However, in late gestation, any such effect was no longer apparent. We also acknowledge that BMI and parity might influence the levels and activity of RAS enzymes as well as Ang(1-7). There were however no significant correlations between BMI and ACE, ACE2 levels, ACE2 activity, and Ang-(1-7) (data not shown).

This is the first study to measure plasma ACE2 in SGA pregnancies. ACE2 mRNA is present in almost all human tissues in particular in the kidney, heart, lung, testis and gastrointestinal tissues, where it has critical regulatory functions (Harmer et al., 2002). Indeed, ACE2 metabolizes Ang II and reduces its levels and produces the vasodilator peptide, Ang-(1-7). Therefore, it is critical for regulating the actions of Ang II mediated by its $\mathrm{AT}_{1} \mathrm{R}$. A previous study from our group showed that placental ACE2 mRNA expression was lower in pregnancies associated with fetal growth restriction (Delforce et al., 2019), suggesting that reduced placental ACE2 levels and reduced Ang-(1-7) production might contribute to the etiology of SGA. The elevation in plasma ACE2 levels in SGA, taken together with the reduction in placental ACE2, suggests, however, that placental ACE2 is not contributing to the high levels of sACE2 in the maternal circulation in SGA pregnancies. Other tissues that could contribute ACE2 to the maternal circulation include the kidney, which has high levels of ACE2 and a 50\% increase in renal blood flow during pregnancy (Brosnihan et al., 2003).

Further studies are required to investigate the relationships between placental ACE and ACE2 mRNA expression and activity and plasma ACE and ACE2 levels and activity in matched samples from SGA pregnancies to fully elucidate the effects of these two enzymes in the etiology of SGA. These studies could also be used to determine if ACE and ACE2 are early biomarkers for SGA or fetal growth restriction (see Zhou et al., 2013).

Samples from women with PE were collected from two different locations in Australia (New South Wales and South Australia). Although the methods of sample collection were very similar at the 2 sites we acknowledge that it could have influenced the levels of ACE2, ACE, NEP and Ang-(1-7). However, the clinical characteristics of these PE subgroups were similar (Supplementary Table 1) and there were no significant differences in levels of RAS enzymes and Ang peptides in samples collected from these two sites (Supplementary Figure 1). We also showed that there were no significant changes in levels/activity of RAS enzymes and Ang-(1-7) across gestation in women with PE (Supplementary Figure 2). Another limitation of the study is that in some cases samples were collected before the onset of PE and, in other cases, women may have been taking antihypertensive medications to control blood pressure. Both of these factors could influence the levels and activity of RAS components.
Unfortunately, we do not have access to BP measurements from women after PE was diagnosed nor information on medications taken by the women. Therefore, some degree of caution should be taken in interpreting our findings.

Women with PE were found to have lower ACE and ACE2 (levels and activity) than women with normal pregnancies. This decrease in the angiotensin processing enzymes would be expected to result in altered Ang peptide levels in women with PE. We found that Ang-(1-7) levels were reduced in women with PE compared with women with normal pregnancies, and it is well known that levels of Ang II are suppressed in PE (Merrill et al., 2002; Velloso et al., 2007; Brosnihan et al., 2020). The decrease in Ang II could be due to the reduction in ACE and the increased amount of ACE2 relative to ACE (Figure 5F). In addition, we have shown for the first time that there is decreased levels of NEP in women with PE. Decreased levels of both ACE2 as well as NEP could account for the decreased levels of Ang-(1-7) in women with PE.

Importantly, some of the women in the PE group delivered SGA babies. In the SGA group, ACE2 was elevated in early gestation but in the PE group, maternal circulating ACE2 levels and activity were suppressed at term. This could be due to the timing of sampling (early gestation vs. term), since we have previously shown that Ang-(1-7) levels are similarly increased at 15 weeks of gestation in women who go on to develop preeclampsia (Sykes et al., 2014a), whereas at term Ang-(1-7) levels are reduced.

ACE2 protects tissues from the pro-inflammatory effects of Ang II by metabolizing it and producing Ang-(1-7) (Jia, 2016). As well, ACE2 is the receptor for SARS-CoV-2. Ang II has proinflammatory effects similar to those caused by the SARS virus. In acute respiratory distress syndrome recombinant human ACE2 reduces SARS-induced lung injury (Imai et al., 2005) and has been shown to prevent cell infection by SARS-Cov-2 (Monteil et al., 2020; Tai et al., 2020). Therefore, the high levels of sACE2 seen in normal pregnant women might protect the lungs from SARS-CoV-2 induced lung injury and slow down viral entry into cells thus reducing viral spread (Turner et al., 2004; Zhang et al., 2020). Conversely, the low levels of sACE2 found in pregnant women with PE might increase their susceptibility to COVID-19.

\section{CONCLUSION}

In conclusion, our findings on the relationships between ACE and ACE2 in normal pregnancy and pregnancies associated with PE or SGA show that dysfunctional relationships between these two key enzymes in the circulating RAS could be involved in the pathogenesis of PE and SGA. Furthermore, increased levels of sACE2 in pregnant women could be significant in understanding the clinical effects of COVID-19.

\section{DATA AVAILABILITY STATEMENT}

The raw data supporting the conclusions of this article will be made available by the authors, without undue reservation. 


\section{ETHICS STATEMENT}

The studies involving human participants were reviewed and approved by the Queen Elizabeth Hospital and Lyell McEwin Hospital Human Research Ethics Committee, the University of Adelaide Human Research Ethics Committee, the University of Newcastle Research Ethics Committee, and the Hunter New England Health Human Research Ethics Committee. The patients/participants provided their written informed consent to participate in this study.

\section{AUTHOR CONTRIBUTIONS}

ST substantially contributed to the design of the study, acquisition of data, analysis and interpretation of data, revision of the draft article for content, and the final approval of the version submitted for publication. VC and KR provided plasma samples and contributed to the revision of the draft article for content and final approval of the version submitted for publication. DH contributed to the interpretation of the data, revision of the drafted article, and the final approval of the version to be published. EL substantially contributed

\section{REFERENCES}

Brosnihan, K. B., Merrill, D. C., Yamaleyeva, L. M., Chen, K., Neves, L., Joyner, J., et al. (2020). Longitudinal study of angiotensin peptides in normal and preeclamptic pregnancy. Endocrine 69, 410-419. doi: 10.1007/s12020-020-022963

Brosnihan, K. B., Neves, L. A., Joyner, J., Averill, D. B., Chappell, M. C., Sarao, R., et al. (2003). Enhanced renal immunocytochemical expression of ANG-(1-7) and ACE2 during pregnancy. Hypertension 42, 749-753. doi: 10.1161/01.Hyp. 0000085220.53285 .11

Chhabra, K. H., Chodavarapu, H., and Lazartigues, E. (2013). Angiotensin converting enzyme 2: a new important player in the regulation of glycemia. IUBMB Life 65, 731-738. doi: 10.1002/iub.1190

Delforce, S. J., Lumbers, E. R., Ellery, S. J., Murthi, P., and Pringle, K. (2019). Dysregulation of the placental renin-angiotensin system in human fetal growth restriction. Reproduction 158, 237-245. doi: 10.1530/rep-18-0633

Dickinson, H., Davies-Tuck, M., Ellery, S. J., Grieger, J. A., Wallace, E. M., Snow, R. J., et al. (2016). Maternal creatine in pregnancy: a retrospective cohort study. BJOG 123, 1830-1838. doi: 10.1111/1471-0528.14237

Donoghue, M., Hsieh, F., Baronas, E., Godbout, K., Gosselin, M., Stagliano, N., et al. (2000). A novel angiotensin-converting enzyme-related carboxypeptidase (ACE2) converts angiotensin I to angiotensin 1-9. Circ. Res. 87, E1-E9.

Escobar, E., Rodriguez-Reyna, T. S., Arrieta, O., and Sotelo, J. (2004). Angiotensin II, cell proliferation and angiogenesis regulator: biologic and therapeutic implications in cancer. Curr. Vasc. Pharmacol. 2, 385-399. doi: 10.2174/ 1570161043385556

Gallagher, P. E., Ferrario, C. M., and Tallant, E. A. (2008). MAP kinase/phosphatase pathway mediates the regulation of ACE2 by angiotensin peptides. Am. J. Physiol. Cell Physiol. 295, C1169-C1174. doi: 10.1152/ajpcell.00145. 2008

Harmer, D., Gilbert, M., Borman, R., and Clark, K. L. (2002). Quantitative mRNA expression profiling of ACE 2, a novel homologue of angiotensin converting enzyme. FEBS Lett. 532, 107-110. doi: 10.1016/s0014-5793(02)03640-2

Imai, Y., Kuba, K., Rao, S., Huan, Y., Guo, F., Guan, B., et al. (2005). Angiotensinconverting enzyme 2 protects from severe acute lung failure. Nature 436, 112-116. doi: 10.1038/nature03712

Irani, R. A., and Xia, Y. (2008). The functional role of the renin-angiotensin system in pregnancy and preeclampsia. Placenta 29, 763-771. doi: 10.1016/j.placenta. 2008.06.011 to the interpretation of data, article drafting and revision for important intellectual content, and the final approval of the version to be published. KP substantially contributed to the design of the study, analysis and interpretation of data, revision of the draft article for content, and the final approval of the version submitted for publication. All authors contributed to the article and approved the submitted version.

\section{FUNDING}

KP was supported by an Australian Research Council (ARC) Future Fellowship. VC was supported by a National Health and Medical Research Council (NHMRC) Senior Research Fellowship.

\section{SUPPLEMENTARY MATERIAL}

The Supplementary Material for this article can be found online at: https://www.frontiersin.org/articles/10.3389/fphys. 2020.590787/full\#supplementary-material

Jia, H. (2016). Pulmonary angiotensin-converting enzyme 2 (ACE2) and inflammatory lung disease. Shock 46, 239-248. doi: 10.1097/shk. 0000000000000633

Lambert, D. W., Yarski, M., Warner, F. J., Thornhill, P., Parkin, E. T., Smith, A. I., et al. (2005). Tumor necrosis factor- $\alpha$ convertase (ADAM17) mediates regulated ectodomain shedding of the severe-acute respiratory syndrome-coronavirus (SARS-CoV) receptor, angiotensin-converting enzyme2 (ACE2). J. Biol. Chem. 280, 30113-30119. doi: 10.1074/jbc.m5051 11200

Lew, R. A., Warner, F. J., Hanchapola, I., Yarski, M. A., Ramchand, J., Burrell, L. M., et al. (2008). Angiotensin-converting enzyme 2 catalytic activity in human plasma is masked by an endogenous inhibitor. Exp. Physiol. 93, 685-693. doi: 10.1113/expphysiol.2007.040352

Lowe, S. A., Bowyer, L., Lust, K., McMahon, L. P., Morton, M., North, R. A., et al (2015). SOMANZ guidelines for the management of hypertensive disorders of pregnancy 2014. Aust. N. Z. J. Obstet. Gynaecol. 55, e1-e29. doi: 10.1111/ajo. 12399

Lumbers, E. R., and Pringle, K. G. (2014). Roles of the circulating reninangiotensin-aldosterone system in human pregnancy. Am. J. Physiol. Regul. Integr. Comp. Physiol. 306, R91-R101. doi: 10.1152/ajpregu.00034.2013

Malard, L., Kakinami, L., O’Loughlin, J., Roy-Gagnon, M.-H., Labbe, A., Pilote, L., et al. (2013). The association between the angiotensin-converting enzyme2 gene and blood pressure in a cohort study of adolescents. BMC Med. Genet. 14:117. doi: 10.1186/1471-2350-14-117

Merrill, D. C., Karoly, M., Chen, K., Ferrario, C. M., and Brosnihan, K. B. (2002). Angiotensin-(1-7) in normal and preeclamptic pregnancy. Endocrine 18, 239-245. doi: 10.1385/endo 18, 239

Monteil, V., Kwon, H., Prado, P., Hagelkrüys, A., Wimmer, R. A., Stahl, M., et al. (2020). Inhibition of SARS-CoV-2 infections in engineered human tissues using clinical-grade soluble human ACE2. Cell 181, 905.e-913.e7. doi: 10.1016/j.cell. 2020.04.004

Oudit, G. Y., Imai, Y., Kuba, K., Scholey, J. W., and Penninger, J. M. (2009). The Role of ACE2 in Pulmonary Diseases-Relevance for the Nephrologist. Oxford: Oxford University Press.

Patnaik, M., Pati, P., Swain, S. N., Mohapatra, M. K., Dwibedi, B., Kar, S. K., et al. (2014). Association of angiotensin-converting enzyme and angiotensinconverting enzyme-2 gene polymorphisms with essential hypertension in the population of Odisha. India. Ann. Hum. Biol. 41, 145-152. doi: 10.3109/ 03014460.2013 .837195 
Shao, Z., Shrestha, K., Borowski, A. G., Kennedy, D. J., Epelman, S., Thomas, J. D., et al. (2013). Increasing serum soluble angiotensin-converting enzyme 2 activity after intensive medical therapy is associated with better prognosis in acute decompensated heart failure. J. Card. Fail. 19, 605-610. doi: 10.1016/j. cardfail.2013.06.296

Sykes, S. D., Pringle, K. G., Zhou, A., Dekker, G. A., Roberts, C. T., Lumbers, E. R., et al. (2014a). Fetal sex and the circulating renin-angiotensin system during early gestation in women who later develop preeclampsia or gestational hypertension. J. Hum. Hypertens. 28, 133-139. doi: 10.1038/jhh. 2013.51

Sykes, S. D., Pringle, K. G., Zhou, A., Dekker, G. A., Roberts, C. T., and Lumbers, E. R. (2014b). The balance between human maternal plasma angiotensin II and angiotensin 1-7 levels in early gestation pregnancy is influenced by fetal sex. J. Renin Angiotensin Aldosterone Syst. 15, 523-531. doi: 10.1177/ 1470320313477174

Tai, W., He, L., Zhang, X., Pu, J., Voronin, D., Jiang, S., et al. (2020). Characterization of the receptor-binding domain (RBD) of 2019 novel coronavirus: implication for development of RBD protein as a viral attachment inhibitor and vaccine. Cell. Mol. Immunol. 17, 613-620. doi: 10.1038/s41423020-0400-4

Turner, A. J., Hiscox, J. A., and Hooper, N. M. (2004). ACE2: from vasopeptidase to SARS virus receptor. Trends Pharmacol. Sci. 25, 291-294. doi: 10.1016/j.tips. 2004.04.001

Velloso, E. P., Vieira, R., Cabral, A. C., Kalapothakis, E., and Santos, R. A. S. (2007). Reduced plasma levels of angiotensin-(1-7) and renin activity in preeclamptic patients are associated with the angiotensin I- converting enzyme deletion/deletion genotype. Braz. J. Med. Biol. Res. 40, 583-590. doi: 10.1590/ s0100-879x2007000400018

Vickers, C., Hales, P., Kaushik, V., Dick, L., Gavin, J., Tang, J., et al. (2002). Hydrolysis of biological peptides by human angiotensin-converting enzymerelated carboxypeptidase. J. Biol. Chem. 277, 14838-14843. doi: 10.1074/jbc. M200581200

Walls, A. C., Park, Y. J., Tortorici, M. A., Wall, A., McGuire, A. T., and Veesler, D. (2020). Structure, function, and antigenicity of the SARSCoV-2 spike glycoprotein. Cell 181, 281.e6-292.e6. doi: 10.1016/j.cell.2020. 02.058

Wan, Y., Shang, J., Graham, R., Baric, R. S., and Li, F. (2020). Receptor recognition by the novel coronavirus from wuhan: an analysis based on decade-long structural studies of SARS coronavirus. J. Virol. 94:e00127-20. doi: 10.1128/jvi. 00127-20
Wang, L. P., Fan, S. J., Li, S. M., Wang, X. J., Gao, J. L., and Yang, X. H. (2016). Protective role of ACE2-Ang-(1-7)-Mas in myocardial fibrosis by downregulating KCa3.1 channel via ERK1/2 pathway. Pflugers Arch. 468, 2041 2051. doi: 10.1007/s00424-016-1875-9

Warner, F. J., Smith, A. I., Hooper, N. M., and Turner, A. J. (2004). Angiotensinconverting enzyme-2: a molecular and cellular perspective. Cell Mol. Life. Sci. 61, 2704-2713. doi: 10.1007/s00018-004-4240-7

Wrapp, D., Wang, N., Corbett, K. S., Goldsmith, J. A., Hsieh, C. L., Abiona, O., et al. (2020). Cryo-EM structure of the 2019-nCoV spike in the prefusion conformation. Science 367, 1260-1263. doi: 10.1126/science. abb2507

Xiao, F., and Burns, K. D. (2017). Measurement of angiotensin converting enzyme 2 activity in biological fluid (ACE2). Methods Mol. Biol. 1527, 101-115. doi: 10.1007/978-1-4939-6625-7_8

Yamaleyeva, L. M., Pulgar, V. M., Lindsey, S. H., Yamane, L., Varagic, J., McGee, C., et al. (2015). Uterine artery dysfunction in pregnant ACE2 knockout mice is associated with placental hypoxia and reduced umbilical blood flow velocity. Am. J. Physiol. Endocrinol. Metab. 309, E84-E94. doi: 10.1152/ajpendo.00596. 2014

Zhang, H., Penninger, J. M., Li, Y., Zhong, N., and Slutsky, A. S. (2020). Angiotensin-converting enzyme 2 (ACE2) as a SARS-CoV-2 receptor: molecular mechanisms and potential therapeutic target. Intensive Care Med. 46, 586-590. doi: 10.1007/s00134-020-05985-9

Zhou, A., Dekker, G. A., Lumbers, E. R., Leemaqz, S. Y., Thompson, S. D., and Heinemann, G. (2013). The association of maternal ACE A11860G with small for gestational age babies is modulated by the environment and by fetal sex: a multicentre prospective case-control study. Mol. Hum. Reprod. 19, 618-627. doi: 10.1093/molehr/gat029

Conflict of Interest: The authors declare that the research was conducted in the absence of any commercial or financial relationships that could be construed as a potential conflict of interest.

Copyright (C) 2020 Tamanna, Clifton, Rae, van Helden, Lumbers and Pringle. This is an open-access article distributed under the terms of the Creative Commons Attribution License (CC BY). The use, distribution or reproduction in other forums is permitted, provided the original author(s) and the copyright owner(s) are credited and that the original publication in this journal is cited, in accordance with accepted academic practice. No use, distribution or reproduction is permitted which does not comply with these terms. 